\title{
Microbial reduction of a $U(V)$ complex with an organic ligand
}

\author{
MARGAUX MOLINAS ${ }^{1}$, RADMILA FAIZOVA ${ }^{2}$, ASHLEY \\ BROWN $^{2}$, KARIN LEDERBALLE MEIBOM ${ }^{3}$, TIM \\ PRUESSMANN $^{4}$, TONYA VITOVA ${ }^{4}$, MARINELLA $^{2}$ \\ MAZZANTI $^{2}$ AND RIZLAN BERNIER-LATMANI ${ }^{2}$ \\ ${ }^{1}$ EPFL ENAC IIE EML \\ ${ }^{2} \mathrm{EPFL}$ \\ ${ }^{3}$ Ecole Polytechnique Federale de Lausanne \\ ${ }^{4} \mathrm{KIT}$ \\ Presenting Author: margaux.molinas@epfl.ch
}

The molecular mechanism of hexavalent uranium (U(VI)) reduction to tetravalent (U(IV)) species remains partly unresolved for metal-reducing bacteria such as Shewanella oneidensis MR-1. All evidence points to the first step being the one-electron reduction of $U(V I)$ to $U(V)^{1,2}$. We confirm that finding by capturing the $U(V)$ intermediate using the unusual properties of the aminocarboxylate ligand, dpaea ${ }^{3}$, which forms a stable aqueous complex with $\mathrm{U}(\mathrm{V})$ at $\mathrm{pH} 7$. Dpaea provides the opportunity to directly investigate the fate of the U(V) species. Previous work has attributed U(IV) formation to the disproportionation of $2 \mathrm{U}(\mathrm{V})$ molecules to $\mathrm{U}(\mathrm{VI})$ and $\mathrm{U}(\mathrm{IV})$ when carbonate was the ligand ${ }^{1,2}$. However, the elucidation of this step requires more direct experimental evidence for other ligands. Here, we investigate the fate of $U(V)$-dpaea in the presence of $S$. oneidensis MR-1 by combining ion exchange chromatography, UV-vis spectrometry, and U M4-edge High Resolution X-ray Absorption Near Edge Structure spectroscopy. On the one hand, we probed the ability of wild-type and mutant strains of $S$. oneidensis MR-1 to interact with U(V)-dpaea. These mutant strains lacked part or all of their $c$-type cytochromes, proteins key to electron tranfer. On the other hand, we reacted $\mathrm{U}(\mathrm{V})$-dpaea with purified outer-membrane $c$-type cytochrome MtrC, a terminal reductase. The combination of these two systems allowed us (i) to rule out disproportionation as a major mechanism for $\mathrm{U}(\mathrm{V})$-dpaea transformation to U(IV), and (ii) to deduce that $\mathrm{U}(\mathrm{V})$-dpaea is biologically reduced to $\mathrm{U}(\mathrm{IV})$ via an active electron transfer mediated by $c$-type cytochromes. Altogether, these results suggest that $\mathrm{U}(\mathrm{V})$ could be biologically reduced when bound to a stabilizing organic ligand. 